\title{
Application of Adomian decomposition method to solving some kinds of partial differential equations and system of partial differential equations
}

\author{
Justin Mouyedo Loufouilou ${ }^{2}$, Gabriel Bissanga ${ }^{2}$, Bassono Francis ${ }^{1}$, Pare Youssouf ${ }^{1 *}$ \\ ${ }^{1}$ University of Ouagadougou \\ ${ }^{2}$ University Marien Ngouabi \\ *Corresponding author E-mail: pareyoussouf@yahoo.fr
}

\begin{abstract}
In this article, the Adomian decomposition method (ADM) is used to construct the solution of the initial value problem of some kinds of partial differential equations of first order.
\end{abstract}

AMS Subject Classification: 47H14; 34G20; 47J25; 65J15.

Keywords: Adomian decomposition method, partial differential equations.

\section{Introduction}

In the last years, the ADM has been applied to get the solutions of various kinds of problems of ODEs and PDEs [1][11]. Here, we use the Adomian decomposition method, to investigate partial differential equations and system of partial differential equations of first order. Using this last method, we get the exact solution.

\section{The adomian decomposition method}

\subsection{About the adomian decomposition method [1], [2], [3], [4], [5]}

Suppose that we need to solve the following equation:

$$
A u=f
$$

In a real Hilbert space $\mathrm{H}$, where $A: H \rightarrow H$ a linear is or a nonlinear operator, $f \in H$ and $u$ is the unknown. The principle of the ADM is based on the decomposition of the nonlinear operator $A$ in the following form:

$$
A=L+R+N
$$

Where $L+R$ is linear, $N$ nonlinear, $L$ invertible with $L^{-1}$ as inverse. Using that decomposition, equation (2.1) is equivalent to

$$
u=\theta+L^{-1} f-L^{-1} R u-L^{-1} N u
$$

Where $\theta$ verifies $L \theta=0$. (2.2) is called the Adomian's fundamental equation or Adomian's canonical form. We look for the solution of (2.1) in a series expansion form $u=\sum_{n=0}^{+\infty} u_{n}$ and we consider $N u=\sum_{n=0}^{+\infty} A_{n}$ where $A_{n}$ are special polynomials of variables $u_{0}, u_{1}, \ldots, u_{n}$ called Adomian polynomials and defined by [1], [2], [3], [4]:

$$
A_{n}=\frac{1}{n !} \frac{d^{n}}{d \lambda^{n}}\left[N\left(\sum_{i=0}^{+\infty} \lambda^{i} u_{i}\right)\right]_{\lambda=0} \quad n=0,1,2, \ldots
$$

Where $\lambda$ is a parameter used by "convenience". Thus (2.2) can be rewritten as follows:

$$
\sum_{n=0}^{+\infty} u_{n}=\theta+L^{-1} f-L^{-1} R\left(\sum_{n=0}^{+\infty} u_{n}\right)-L^{-1}\left(\sum_{n=0}^{+\infty} A_{n}\right)
$$


We suppose that the series $\sum_{n=0}^{+\infty} u_{n}$ and $\sum_{n=0}^{+\infty} A_{n}$ are convergent, and obtain by identification the Adomian algorithm:

$$
\left\{\begin{array}{c}
u_{0}=\theta+L^{-1} f \\
u_{1}=-L^{-1}\left(R u_{0}\right)-L^{-1} A_{0} \\
\cdots \\
u_{n+1}=-L^{-1}\left(R u_{n}\right)-L^{-1} A_{n}
\end{array}\right.
$$

In practice, it is often difficult to calculate all terms of an Adomian series, so we approach the series solution by the truncated series: $u=\sum_{i=0}^{n} u_{i}$, where the choice of $n$ depends on error requirements.

\section{Applications}

\subsection{Example 1}

Using the Adomian decomposition method, let's construct the solution of the following partial differential equation:

$$
\left\{\begin{array}{l}
\frac{\partial u(x, y)}{\partial x}+\frac{\partial u(x, y)}{\partial y}=x+y \\
u(x, 0)=0 \\
u(0, y)=0
\end{array}\right.
$$

Where

$$
u(x, y) \in C^{1}(\Omega) ; \Omega \subset[0, T] \times[0, T]
$$

Let's take

$$
\left\{\begin{array}{l}
L_{x}(.)=\frac{\partial(.)}{\partial x} ; L_{x}^{-1}(.)=\int_{0}^{x}(.) d s \\
L_{y}(.)=\frac{\partial(.)}{\partial y} ; L_{y}^{-1}(.)=\int_{0}^{y}(.) d s
\end{array}\right.
$$

From (3.1), we have:

$$
\left\{\begin{array}{l}
\frac{\partial u}{\partial x}=x+y-\frac{\partial u}{\partial y} \\
\frac{\partial u}{\partial y}=x+y-\frac{\partial u}{\partial x}
\end{array}\right.
$$

(3.4) give us:

$$
\left\{\begin{aligned}
u(x, y) & =u(0, y)+\int_{0}^{x}(s+y) d s-\int_{0}^{x} \frac{\partial u(s, y)}{\partial y} d s \\
& =\frac{x^{2}}{2}+x y-\int_{0}^{x} \frac{\partial u(s, x)}{\partial y} d s \\
u(x, y) & =u(x, 0)+\int_{0}^{y}(x+s) d s-\int_{0}^{y} \frac{\partial u(x, s)}{\partial y} d s \\
& =\frac{y^{2}}{2}+x y-\int_{0}^{y} \frac{\partial u(x, s)}{\partial y} d s
\end{aligned}\right.
$$

From (3.5), we obtain:

$$
u(x, y)=\frac{x^{2}}{4}+\frac{y^{2}}{4}+x y-\frac{1}{2} \int_{0}^{x} \frac{\partial u(s, y)}{\partial y} d s-\frac{1}{2} \int_{0}^{y} \frac{\partial u(x, s)}{\partial y} d s
$$


Let us suppose that the solution $u(x, y)$ of the initial value problem (3.1) has the following form:

$$
u(x, y)=\sum_{n=0}^{\infty} u_{n}(x, y)
$$

From (3.6), we have the following Adomian algorithm:

$$
\left\{\begin{array}{l}
u_{0}(x, y)=\frac{x^{2}+y^{2}}{4}+x y \\
u_{n+1}(x, y)=-\frac{1}{2} \int_{0}^{x} \frac{\partial u_{n}(s, y)}{\partial y} d s-\frac{1}{2} \int_{0}^{x} \frac{\partial u_{n}(s, y)}{\partial x} d s ; n \geq 0
\end{array}\right.
$$

which gives us:

$$
\left\{\begin{array}{l}
u_{1}(x, y)=-\frac{x^{2}+y^{2}}{4}-\frac{x y}{2} \\
u_{2}(x, y)=\frac{x^{2}+y^{2}}{8}+\frac{x y}{2} \\
u_{3}(x, y)=-\frac{x^{2}+y^{2}}{8}-\frac{x y}{4} \\
u_{4}(x, y)=\frac{x^{2}+y^{2}}{16}+\frac{x y}{4} \\
u_{5}(x, y)=-\frac{x^{2}+y^{2}}{16}-\frac{x y}{8} \\
\ldots
\end{array}\right.
$$

and further terms can easily be calculated using a symbolic computation package.

Thus the solution of (3.1) is:

$$
\begin{aligned}
u(x, y) & =\sum_{n=0}^{\infty} u_{n}(x, y)=\left(x y+\frac{x^{2}+y^{2}}{4}\right)+\left(-\frac{x^{2}+y^{2}}{4}-\frac{x y}{2}\right)+\left(\frac{x^{2}+y^{2}}{8}+\frac{x y}{2}\right)+ \\
& \left(-\frac{x^{2}+y^{2}}{8}-\frac{x y}{4}\right)+\left(\frac{x^{2}+y^{2}}{16}+\frac{x y}{4}\right)+\left(-\frac{x^{2}+y^{2}}{16}-\frac{x y}{8}\right)+\ldots \\
& =x y
\end{aligned}
$$

\subsection{Example 2}

Let us consider the following initial value problem

$$
\left\{\begin{array}{l}
\frac{\partial u(x, y)}{\partial x}+\frac{\partial u(x, y)}{\partial y}=x^{2}+y^{2}+4 x y \\
u(x, 0)=0 \\
u(0, y)=0
\end{array}\right.
$$

Where

$$
u(x, y) \in C^{1}(\Omega) ; \Omega \subset[0, T] \times[0, T]
$$

Let's take

$$
\left\{\begin{array}{l}
L_{x}(.)=\frac{\partial(.)}{\partial x} ; L_{x}^{-1}(.)=\int_{0}^{x}(.) d s \\
L_{y}(.)=\frac{\partial(.)}{\partial y} ; L_{y}^{-1}(.)=\int_{0}^{y}(.) d s
\end{array}\right.
$$

From (3.10), we have: 


$$
\left\{\begin{array}{l}
\frac{\partial u}{\partial x}=x^{2}+y^{2}+4 x y-\frac{\partial u}{\partial y} \\
\frac{\partial u}{\partial y}=x^{2}+y^{2}+4 x y-\frac{\partial u}{\partial x}
\end{array}\right.
$$

(3.13) give us:

$$
\left\{\begin{array}{l}
u(x, y)=u(0, y)+\int_{0}^{x}\left(s^{2}+4 s y+y^{2}\right) d s-\int_{0}^{x} \frac{\partial u(s, y)}{\partial x} d s \\
\Rightarrow u(x, y)=\frac{x^{3}}{3}+2 x^{2} y+y^{2} x-\int_{0}^{x} \frac{\partial u(s, y)}{\partial x} d s \\
u(x, y)=u(x, 0)+\int_{0}^{y}(x+s) d s-\int_{0}^{y} \frac{\partial u(x, s)}{\partial y} d s \\
\Rightarrow u(x, y)=\frac{y^{3}}{3}+2 x y^{2}+x^{2} y-\int_{0}^{y} \frac{\partial u(x, s)}{\partial y} d s
\end{array}\right.
$$

From (3.14), we obtain:

$$
u(x, y)=\frac{1}{6}\left(x^{3}+y^{3}\right)+\frac{3}{2}\left(y^{2} x+x^{2} y\right)-\frac{1}{2} \int_{0}^{x} \frac{\partial u(s, y)}{\partial x} d s-\frac{1}{2} \int_{0}^{y} \frac{\partial u(x, s)}{\partial y} d s
$$

Let us suppose that the solution $u(x, y)$ of the initial value problem (3.10) has the following form:

$$
u(x, y)=\sum_{n=0}^{\infty} u_{n}(x, y) .
$$

From (3.15), we have the following Adomian algorithm

$$
\left\{\begin{array}{l}
u_{0}(x, y)=\frac{1}{6}\left(x^{3}+y^{3}\right)+\frac{3}{2}\left(y^{2} x+x^{2} y\right) \\
u_{n+1}(x, y)=-\frac{1}{2} \int_{0}^{x} \frac{\partial u_{n}(s, y)}{\partial x} d s-\frac{1}{2} \int_{0}^{y} \frac{\partial u_{n}(x, s)}{\partial y} d s ; n \geq 0
\end{array}\right.
$$

Which gives us:

$$
\left\{\begin{array}{l}
u_{1}(x, y)=-\frac{3}{12}\left(x^{3}+y^{3}\right)-\frac{4}{4}\left(y^{2} x+x^{2} y\right) \\
u_{2}(x, y)=\frac{4}{24}\left(x^{3}+y^{3}\right)+\frac{7}{8}\left(y^{2} x+x^{2} y\right) \\
u_{3}(x, y)=-\frac{7}{48}\left(x^{3}+y^{3}\right)-\frac{11}{16}\left(y^{2} x+x^{2} y\right) \\
u_{4}(x, y)=\frac{11}{96}\left(x^{3}+y^{3}\right)+\frac{18}{32}\left(y^{2} x+x^{2} y\right) \\
u_{5}(x, y)=-\frac{18}{192}\left(x^{3}+y^{3}\right)-\frac{29}{64}\left(y^{2} x+x^{2} y\right) \\
\ldots
\end{array}\right.
$$

and the further terms can easily be calculated using a symbolic computation package.

We notice that:

Where

$$
u_{n}(x, y)=v_{n}(x, y)+w_{n}(x, y)
$$

$$
\begin{aligned}
& v_{n}(x, y)=\frac{(-1)^{n}}{3 \cdot 2^{n+1}} t^{n} \times A(x, y) \\
& w_{n}(x, y)=\frac{(-1)^{n}}{2^{n+1}} l_{n} \times B(x, y) \\
& A(x, y)=x^{3}+y^{3} \\
& B(x, y)=y^{2} x+x^{2} y
\end{aligned}
$$


$\left(t_{n}\right)$ And $\left(l_{n}\right)$ are the series which satisfy the following relations:

$$
\begin{aligned}
& \left\{\begin{array}{l}
t_{n+2}-t_{n+1}=t_{n} \\
t_{0}=1 \\
t_{1}=4
\end{array}\right. \\
& \left\{\begin{array}{l}
l_{n+2}-l_{n+1}=l_{n} \\
l_{0}=3 \\
l_{1}=4
\end{array}\right.
\end{aligned}
$$

The solutions of (3.21) and (3.22) are respectively:

$$
t_{n}=\left(\frac{1-\sqrt{5}}{2}\right)^{n+1}+\left(\frac{1+\sqrt{5}}{2}\right)^{n+1}
$$

and

$$
l_{n}=\frac{3-\sqrt{5}}{2}\left(\frac{1-\sqrt{5}}{2}\right)^{n}+\frac{3+\sqrt{5}}{2}\left(\frac{1+\sqrt{5}}{2}\right)^{n}
$$

thus

$$
\begin{aligned}
& v_{n}(x, y)=\frac{(-1)^{n} A_{n}(x, y)}{3}\left[\frac{(1-\sqrt{5})^{n+1}}{2^{2 n+2}}+\frac{(1+\sqrt{5})^{n+1}}{2^{2 n+2}}\right] \\
& w_{n}(x, y)=(-1)^{n} B(x, y)\left[(3-\sqrt{5}) \frac{(1-\sqrt{5})^{n}}{2^{2 n+2}}+(3+\sqrt{5}) \frac{(1+\sqrt{5})^{n}}{2^{2 n+2}}\right]
\end{aligned}
$$

The solution of (3.10) is:

$$
\begin{aligned}
u(x, y) & =\sum_{n=0}^{+\infty} u_{n}(x, y)=\sum_{n=0}^{+\infty}\left(u_{2 n}(x, y)+u_{2 n+1}(x, y)\right) \\
& =\sum_{n=0}^{+\infty}\left[\left(v_{2 n}(x, y)+w_{2 n}(x, y)\right)+\left(\left(v_{2 n+1}(x, y)+w_{2 n+1}(x, y)\right)\right]\right.
\end{aligned}
$$

We notice that:

$$
\begin{aligned}
& \sum_{n=0}^{+\infty} v_{2 n}(x, y)=\frac{A(x, y)}{3}\left[\sum_{n=0}^{+\infty} \frac{(1-\sqrt{5})^{2 n+1}}{2^{4 n+2}}+\sum_{n=0}^{+\infty} \frac{(1+\sqrt{5})^{2 n+1}}{2^{4 n+2}}\right] \\
& =\frac{A(x, y)}{3}\left[\frac{1-\sqrt{5}}{4} \sum_{n=0}^{+\infty}\left[\left(\frac{1-\sqrt{5}}{4}\right)^{2}\right]^{n}+\frac{1+\sqrt{5}}{4} \sum_{n=0}^{+\infty}\left[\left(\frac{1+\sqrt{5}}{4}\right)^{2}\right]^{n}\right] \\
& =\frac{A(x, y)}{3}\left\{\frac{1-\sqrt{5}}{4} \lim _{n \rightarrow+\infty} \frac{1-\left[\left(\frac{1-\sqrt{5}}{4}\right)^{2}\right]^{n}}{1-\left(\frac{1-\sqrt{5}}{4}\right)^{2}}+\frac{1+\sqrt{5}}{4} \lim _{n \rightarrow+\infty} \frac{1-\left[\left(\frac{1+\sqrt{5}}{4}\right)^{2}\right]^{n}}{1-\left(\frac{1+\sqrt{5}}{4}\right)^{2}}\right\} \\
& =\frac{A(x, y)}{3}\left\{\frac{1-\sqrt{5}}{4} \times \frac{1}{1-\left(\frac{1-\sqrt{5}}{4}\right)^{2}}+\frac{1+\sqrt{5}}{4} \times \frac{1}{1-\left(\frac{1+\sqrt{5}}{4}\right)^{2}}\right\} \\
& =\frac{A(x, y)}{3}\left(\frac{5-3 \sqrt{5}}{5}+\frac{5+3 \sqrt{5}}{5}\right)=\frac{2 A(x, y)}{3}=\frac{2}{3}\left(x^{3}+y^{3}\right)
\end{aligned}
$$

We obtain: 


$$
\begin{aligned}
& \sum_{n=0}^{+\infty} v_{2 n}(x, y)=\frac{2}{3}\left(x^{3}+y^{3}\right) \\
& \sum_{n=0}^{+\infty} w_{2 n}(x, y)=B(x, y)\left[(3-\sqrt{5}) \sum_{n=0}^{+\infty} \frac{(1-\sqrt{5})^{2 n}}{2^{4 n+2}}+(3+\sqrt{5}) \sum_{n=0}^{+\infty} \frac{(1+\sqrt{5})^{2 n}}{2^{4 n+2}}\right] \\
& =B(x, y)\left[\frac{3-\sqrt{5}}{4} \sum_{n=0}^{+\infty}\left[\left(\frac{1-\sqrt{5}}{4}\right)^{2}\right]^{n}+\frac{3+\sqrt{5}}{4} \sum_{n=0}^{+\infty}\left[\left(\frac{1+\sqrt{5}}{4}\right)^{2}\right]^{n}\right] \\
& =B(x, y)\left\{\frac{3-\sqrt{5}}{4} \lim _{n \rightarrow+\infty} \frac{1-\left[\left(\frac{1-\sqrt{5}}{4}\right)^{2}\right]^{n+1}}{1-\left(\frac{1-\sqrt{5}}{4}\right)^{2}}+\frac{3+\sqrt{5}}{4} \lim _{n \rightarrow+\infty} \frac{1-\left[\left(\frac{1+\sqrt{5}}{4}\right)^{2}\right]^{n+1}}{1-\left(\frac{1+\sqrt{5}}{4}\right)^{2}}\right\} \\
& =B(x, y)\left\{\frac{3-\sqrt{5}}{4} \times \frac{1}{1-\left(\frac{1-\sqrt{5}}{4}\right)^{2}}+\frac{3+\sqrt{5}}{4} \times \frac{1}{1-\left(\frac{1+\sqrt{5}}{4}\right)^{2}}\right\} \\
& =B(x, y)\left(2 \times \frac{5-3 \sqrt{5}}{5}+2 \times \frac{5+3 \sqrt{5}}{5}\right)=4 B(x, y)=4\left(y^{2} x+x^{2} y\right)
\end{aligned}
$$

We obtain:

$$
\begin{aligned}
& \sum_{n=0}^{+\infty} w_{2 n}(x, y)=4\left(y^{2} x+x^{2} y\right) \\
& \sum_{n=0}^{+\infty} v_{2 n+1}(x, y)=-\frac{A(x, y)}{3}\left[\sum_{n=0}^{+\infty} \frac{(1-\sqrt{5})^{2 n+2}}{2^{4 n+4}}+\sum_{n=0}^{+\infty} \frac{(1+\sqrt{5})^{2 n+2}}{2^{4 n+4}}\right] \\
& =-\frac{A(x, y)}{3}\left[\left(\frac{1-\sqrt{5}}{4}\right)^{2} \sum_{n=0}^{+\infty}\left[\left(\frac{1-\sqrt{5}}{4}\right)^{2}\right]^{n}+\left(\frac{1+\sqrt{5}}{4}\right)^{2} \sum_{n=0}^{+\infty}\left[\left(\frac{1+\sqrt{5}}{4}\right)^{2}\right]^{n}\right] \\
& =-\frac{A(x, y)}{3}\left\{\left(\frac{1-\sqrt{5}}{4}\right)^{2} \lim _{n \rightarrow+\infty}\left(\frac{1-\left[\left(\frac{1-\sqrt{5}}{4}\right)^{2}\right]^{n}}{1-\left(\frac{1-\sqrt{5}}{4}\right)^{2}}\right)+\left(\frac{1+\sqrt{5}}{4}\right)^{2} \lim _{n \rightarrow+\infty}\left(\frac{1-\left[\left(\frac{1+\sqrt{5}}{4}\right)^{2}\right]^{n}}{1-\left(\frac{1+\sqrt{5}}{4}\right)^{2}}\right]\right\} \\
& =-\frac{A(x, y)}{3}\left\{\left(\frac{1-\sqrt{5}}{4}\right)^{2} \times \frac{1}{1-\left(\frac{1-\sqrt{5}}{4}\right)^{2}}+\left(\frac{1+\sqrt{5}}{4}\right)^{2} \times \frac{1}{1-\left(\frac{1+\sqrt{5}}{4}\right)^{2}}\right\} \\
& =-\frac{A(x, y)}{3}\left(\frac{5-3 \sqrt{5}}{5}+\frac{5+3 \sqrt{5}}{5}\right)=-\frac{2 A(x, y)}{3}=-\frac{2}{3}\left(x^{3}+y^{3}\right) \\
& \sum_{n=0}^{+\infty} v_{2 n+1}(x, y)=-\frac{2}{3}\left(x^{3}+y^{3}\right)
\end{aligned}
$$




$$
\begin{aligned}
& \sum_{n=0}^{+\infty} w_{2 n+1}(x, y)=-B(x, y)\left[(3-\sqrt{5}) \sum_{n=0}^{+\infty} \frac{(1-\sqrt{5})^{2 n+1}}{2^{4 n+4}}+(3+\sqrt{5}) \sum_{n=0}^{+\infty} \frac{(1+\sqrt{5})^{2 n+1}}{2^{4 n+4}}\right] \\
& =-B(x, y)\left[\frac{(3-\sqrt{5})(1-\sqrt{5})}{16} \sum_{n=0}^{+\infty}\left[\left(\frac{1-\sqrt{5}}{4}\right)^{2}\right]^{n}+\frac{(3+\sqrt{5})(1+\sqrt{5})}{16} \sum_{n=0}^{+\infty}\left[\left(\frac{1+\sqrt{5}}{4}\right)^{2}\right]^{n}\right] \\
& =-B(x, y)\left\{\frac{(3-\sqrt{5})(1-\sqrt{5})}{4} \lim _{n \rightarrow+\infty}\left(\frac{1-\left[\left(\frac{1-\sqrt{5}}{4}\right)^{2}\right]^{n+1}}{1-\left(\frac{1-\sqrt{5}}{4}\right)^{2}}\right)\right\}+ \\
& B(x, y)\left\{\frac{(3+\sqrt{5})(1+\sqrt{5})}{4} \lim _{n \rightarrow+\infty}\left(\frac{1-\left[\left(\frac{1+\sqrt{5}}{4}\right)^{2}\right]^{n+1}}{1-\left(\frac{1+\sqrt{5}}{4}\right)^{2}}\right)\right\} \\
& =-B(x, y)\left\{\frac{(3-\sqrt{5})(1-\sqrt{5})}{4} \times \frac{1}{1-\left(\frac{1-\sqrt{5}}{4}\right)^{2}}+\frac{(3+\sqrt{5})(1+\sqrt{5})}{4} \times \frac{1}{1-\left(\frac{1+\sqrt{5}}{4}\right)^{2}}\right\} \\
& =-B(x, y)\left(\frac{15-7 \sqrt{5}}{10}+\frac{15+7 \sqrt{5}}{10}\right)=-3 B(x, y)=-3\left(y^{2} x+x^{2} y\right) \\
& \sum_{n=0}^{+\infty} w_{2 n+1}(x, y)=-3\left(y^{2} x+x^{2} y\right)
\end{aligned}
$$

Thus the solution of (3.10) is:

$$
u(x, y)=\frac{2}{3}\left(x^{3}+y^{3}\right)+4\left(y^{2} x+x^{2} y\right)-\frac{2}{3}\left(x^{3}+y^{3}\right)-3\left(y^{2} x+x^{2} y\right)=y^{2} x+x^{2} y .
$$

\subsection{Example 3}

Let us consider the following initial value problem

$$
\left\{\begin{array}{l}
\frac{\partial u}{\partial t}+\frac{\partial u}{\partial x}=-2 v \\
\frac{\partial v}{\partial t}+\frac{\partial v}{\partial x}=2 u \\
u(0, x)=\cos x \\
v(0, x)=\sin x
\end{array}\right.
$$

Where

$$
u(t, x) \in C^{1}(\Omega) ; \Omega \subset[0, T] \times[0, T]
$$

Let's take

$$
L_{t}(.)=\frac{\partial(.)}{\partial t} ; L_{x}^{-1}(.)=\int_{0}^{t}(.) d s
$$

From (3.32), we have: 


$$
\begin{aligned}
u(t, y) & =\sum_{n=0}^{+\infty} u_{n}(t, x)=\sum_{n=0}^{+\infty} u_{2 n}(t, x)+\sum_{n=0}^{+\infty} u_{2 n+1}(t, x) \\
& =\left(1-\frac{t^{2}}{2 !}+\frac{t^{4}}{4 !}-\frac{t^{6}}{6 !}+\ldots+(-1)^{n} \frac{t^{2 n}}{(2 n) !}\right) \cos x- \\
& \left(t-\frac{t^{3}}{3 !}+\frac{t^{5}}{5 !}-\frac{t^{7}}{7 !}+\ldots+(-1)^{n} \frac{t^{2 n+1}}{(2 n+1) !}\right) \sin x \\
& =\cos t \cos x-\sin t \sin x
\end{aligned}
$$$$
\left\{\begin{aligned}
u(t, x) & =u(0, x)-\int_{0}^{t} \frac{\partial u(s, x)}{\partial x} d s-2 \int_{0}^{t} v(s, x) d s \\
& =\cos x-\int_{0}^{t} \frac{\partial u(s, x)}{\partial x} d s-2 \int_{0}^{t} v(s, x) d s \\
v(t, x) & =v(0, x)-\int_{0}^{t} \frac{\partial v(s, x)}{\partial x} d s+2 \int_{0}^{t} u(s, x) d s \\
& =\sin x-\int_{0}^{t} \frac{\partial v(s, x)}{\partial x} d s+2 \int_{0}^{t} u(s, x) d s
\end{aligned}\right.
$$

Let us suppose that the solution $u(t, x)$ and $v(t, x)$ of the initial value problem (3.32) has the following form:

$$
\left\{\begin{array}{l}
u(t, x)=\sum_{n=0}^{\infty} u_{n}(t, x) \\
v(t, x)=\sum_{n=0}^{\infty} v_{n}(t, x)
\end{array}\right.
$$

From (3.35), we have the following Adomian algorithm:

$$
\left\{\begin{array}{l}
u_{0}(x, y)=\cos x \\
u_{n+1}(x, y)=-\int_{0}^{t} \frac{\partial u_{n}(s, x)}{\partial x} d s-2 \int_{0}^{t} v_{n}(s, x) d s ; n \geq 0
\end{array}\right.
$$

And

$$
\left\{\begin{array}{l}
v_{0}(x, y)=\sin x \\
v_{n+1}(x, y)=-\int_{0}^{t} \frac{\partial v_{n}(s, x)}{\partial x} d s+2 \int_{0}^{t} u_{n}(s, x) d s ; n \geq 0
\end{array}\right.
$$

(3.37) and (3.38) give us:

$$
\left\{\begin{array}{c}
u_{1}(t, x)=-t \sin x \\
u_{2}(t, x)=-\frac{1}{2 !} t^{2} \cos x \\
u_{3}(t, x)=-\frac{1}{3 !} t^{3} \sin x \\
u_{4}(t, x)=\frac{1}{4 !} t^{4} \cos x \\
\ldots
\end{array}\right.
$$

And

And further terms can easily be calculated using a symbolic computation package. Thus

$$
u(t, x)=\cos (t+x)
$$

And

$$
\left\{\begin{array}{c}
v_{1}(t, x)=t \cos x \\
v_{2}(t, x)=-\frac{1}{2 !} t^{2} \sin x \\
v_{3}(t, x)=-\frac{1}{3 !} t^{3} \cos x \\
v_{4}(t, x)=\frac{1}{4 !} t^{4} \cos x \\
\ldots
\end{array}\right.
$$

$$
\begin{aligned}
v(t, y) & =\sum_{n=0}^{+\infty} v_{n}(t, x)=\sum_{n=0}^{+\infty} v_{2 n}(t, x)+\sum_{n=0}^{+\infty} v_{2 n+1}(t, x) \\
& =\left(1-\frac{t^{2}}{2 !}+\frac{t^{4}}{4 !}-\frac{t^{6}}{6 !}+\ldots+(-1)^{n} \frac{t^{2 n}}{(2 n) !}\right) \sin x+ \\
& \left(t-\frac{t^{3}}{3 !}+\frac{t^{5}}{5 !}-\frac{t^{7}}{7 !}+\ldots+(-1)^{n} \frac{t^{2 n+1}}{(2 n+1) !}+\ldots\right) \cos x \\
& =\cos t \sin x+\sin t \cos x
\end{aligned}
$$




$$
v(t, x)=\sin (t+x)
$$

The solution of (3.32) is:

$$
(u(t, x), v(t, x))=(\cos (t+x), \sin (t+y)) .
$$

\section{Conclusion}

In this paper, we showed once through some examples the useful of the Adomian decomposition method to investigate differential and partial differential equations.

\section{References}

[1] K. ABBAOUI, «Les fondements de la méthode décompositionnelle d'Adomian et application à la résolution de problèmes issus de la biologie et de la médecine. » Thèse de doctorat de l’Université Paris VI. Octobre 1995.

[2] K. ABBAOUI and Y. CHERRUAULT, "Convergence of Adomian method applied to differential equations." Math. Comput. Modelling (28, 5), pp 103-109, 1994.

[3] K. ABBAOUI and Y. CHERRUAULT, "The Decomposition method applied to nonlinear equations". Math. Comput. Modelling (20,9), pp60-73, 1994.

[4] K. ABBAOUI and Y. CHERRUAULT. “The decomposition method applied to the Cauchy problem.” Kybernetes,(28,1),pp 68-74, 1999.

[5] N. NGARASTA, B. SOME, K. ABBAOUI and Y. CHERRUAULT, "New numerical study of Adomian method applied to a diffusion model." Kybernetes, Vol. 31, no1, pp61-75, 2002.

[6] Nafey. A. H. «Perturbation Methods, Wiley, New York, 1973.

[7] Gabriel BISSANGA, A.K NSEMI. " Application of Adomian decomposition method to solving the Van Der Pol equation and comparison with the regular perturbation method." Proceedinds of the Five international workshop on contemporary problems in mathematical physics, Cotonou-Benin,eds. J. Govaerts, M. N. Hounkounou (International Chair in Mathematical Physics and Applications, ICMP-UNESCO Chair, University of Abomey-Calavy,072 BP 50 Cotonou, Republic of Benin, December 2008).

[8] Pierre BAKI-TANGOU, Gabriel BISSANGA, « Application of Adomian decomposition method to solving the Duffing-Van Der Pol equation. Communications in Mathematical Analysis. Vol. 4. No. Pp 30-40(2008).

[9] Chengri Jin, Mingzhu Liu,"A new modification of Adomian decompostion method for solving a kind of evolution equation". Applied Mathematicals and Computation 169(2005) 953-962.

[10] D. Lesnic, "A computational algebraic investigation of the decomposition method for time-dependent problems". Qpplied Mathematics and Computation 119(2001) 197-206.

[11] D. Lesnic, "Blow-up solutions obtained using the decomposition method". Chaos, Solitons 28 (2006) 776-787. 